\title{
Generating Realistic Synthetic Population Datasets
}

\author{
HAO WU, YUE NING, and PRITHWISH CHAKRABORTY, Virginia Tech \\ JILLES VREEKEN, Max Planck Institute for Informatics and Saarland University \\ NIKOLAJ TATTI, Aalto University \\ NAREN RAMAKRISHNAN, Virginia Tech
}

\begin{abstract}
Modern studies of societal phenomena rely on the availability of large datasets capturing attributes and activities of synthetic, city-level, populations. For instance, in epidemiology, synthetic population datasets are necessary to study disease propagation and intervention measures before implementation. In social science, synthetic population datasets are needed to understand how policy decisions might affect preferences and behaviors of individuals. In public health, synthetic population datasets are necessary to capture diagnostic and procedural characteristics of patient records without violating confidentialities of individuals. To generate such datasets over a large set of categorical variables, we propose the use of the maximum entropy principle to formalize a generative model such that in a statistically well-founded way we can optimally utilize given prior information about the data, and are unbiased otherwise. An efficient inference algorithm is designed to estimate the maximum entropy model, and we demonstrate how our approach is adept at estimating underlying data distributions. We evaluate this approach against both simulated data and US census datasets, and demonstrate its feasibility using an epidemic simulation application.
\end{abstract}

\section{CCS Concepts: • Computing methodologies $\rightarrow$ Maximum entropy modeling; $\bullet$ Information systems $\rightarrow$ Data mining;}

Additional Key Words and Phrases: Multivariate categorical data, synthetic population, maximum entropy models, probabilistic modeling

\section{ACM Reference format:}

Hao Wu, Yue Ning, Prithwish Chakraborty, Jilles Vreeken, Nikolaj Tatti, and Naren Ramakrishnan. 2018. Generating Realistic Synthetic Population Datasets. ACM Trans. Knowl. Discov. Data. 12, 4, Article 45 (April 2018), 22 pages.

https://doi.org/10.1145/3182383

This work is supported in part by the Intelligence Advanced Research Projects Activity (IARPA) via Department of Interior National Business Center (DoI/NBC) contract number D12PC000337, by the National Science Foundation via grants DGE1545362, IIS-1633363, and by the Army Research Laboratory under grant W911NF-17-1-0021.

J. Vreeken is supported by the Cluster of Excellence 'Multimodal Computing and Interaction' within the Excellence Initiative of the German Federal Government.

Authors' addresses: H. Wu, Google Inc, Mountain View, CA 94043; email: wuhao723@vt.edu; Y. Ning and N. Ramakrishnan, Discovery Analytics Center, Virginia Tech, Arlington, VA 22203; emails: yning@vt.edu, naren@cs.vt.edu; P. Chakraborty, IBM Watson Health, Cambridge, MA 02142; email: prithwi@vt.edu; J. Vreeken, Max Planck Institute for Informatics and Saarland University, Saarland Informatics Campus, 66123 Saarbrücken, Germany; email: jilles@mpi-inf.mpg.de; N. Tatti, Aalto University School of Science, Department of Information and Computer Science, P.O. Box 15400, FI-00076 Aalto, Finland; email: nikolaj.tatti@aalto.fi.

Permission to make digital or hard copies of all or part of this work for personal or classroom use is granted without fee provided that copies are not made or distributed for profit or commercial advantage and that copies bear this notice and the full citation on the first page. Copyrights for components of this work owned by others than ACM must be honored. Abstracting with credit is permitted. To copy otherwise, or republish, to post on servers or to redistribute to lists, requires prior specific permission and/or a fee. Request permissions from permissions@acm.org.

(C) 2018 ACM 1556-4681/2018/04-ART45 \$15.00

https://doi.org/10.1145/3182383 


\section{INTRODUCTION}

Many research areas, e.g., epidemiology, public health, social science, study the behavior of large populations of individuals under natural scenarios, as well as under human interventions. A key need across these domains is the ready availability of realistic synthetic datasets that can capture key attributes and activities of large populations.

For instance, in epidemiology, synthetic populations are necessary to study disease propagation and intervention measures before implementation. Information from the US census is typically used to model such synthetic datasets. In social science, synthetic populations are necessary to understand how policy decisions might affect preferences and behaviors of individuals. Finally, in public health, synthetic populations are necessary to capture diagnostic and procedural characteristics of patient records without violating confidentialities of individuals.

Typically, the constraints underlying synthetic population generation are assumptions on the supporting marginal or conditional distributions. Although there exist prior studies in estimating probability distributions subject to constraints (e.g., Monte Carlo methods), they are primarily focused on continuous-valued data. Many domains, on the other hand, such as those studied here, feature the need for multi-dimensional categorical datasets.

As a case in point, in epidemiology, one important task is to simulate disease spread and potential outbreaks on the city- or nation-level, and provide useful information to public health officials to support policy and decision making. To make such simulations as accurate as possible, synthetic populations that have the same structural and behavioral properties as the real population are needed. In domains like health care, privacy is an additional issue motivating the design of synthetic populations. In these applications, the necessary datasets to be generated can be represented as tuples with categorical data attributes.

Motivated by these emerging needs, we focus our attention on constructing a generative model that captures given characteristics of categorical population attributes, and best estimates the underlying data generation distribution. However, modeling multi-dimensional categorical data and estimating distributions can be quite challenging due to the exponential possibilities of data spaces in terms of the number of dimensions of categorical data tuples. Although many dimension reduction techniques [29] and pattern recognition algorithms [12, 28] have been proposed and studied in many other machine learning and data mining research areas, it is difficult to simply apply them here in our problem setting. To address these challenges and difficulties, we take the first step here to study this problem. To model categorical data with statistical constraints, we apply the classical and statistically well-founded maximum entropy model. We construct a generative maximum entropy model, which takes the data schema (the set of all categorical attributes that appear in the data) and a set of constraint categorical patterns (a subset of categorical attributes with corresponding probabilities of appearance in the data), as shown in Figure 1, wherein the probabilities of certain categorical patterns are required to satisfy given constraints. In this way, the maximum entropy model maintains the selected characteristics of the underlying categorical data distribution. By sampling the categorical tuples from the maximum entropy model, synthetic population datasets can be generated as illustrated by Figure 1.

Generally, solving maximum entropy models can be infeasible in practice. In this article, we show that by leveraging the structure of the categorical data space in our setting, the maximum entropy model could be inferred quite efficiently. We also propose a heuristic together with the Bayesian information criterion (BIC) to select a simple as well as informative model. To summarize our approach in a nutshell, our contributions are as follows:

(1) We formalize the problem of generating synthetic population datasets via a generative maximum entropy model for categorical data, which captures the statistical features of the underlying categorical data distributions. 


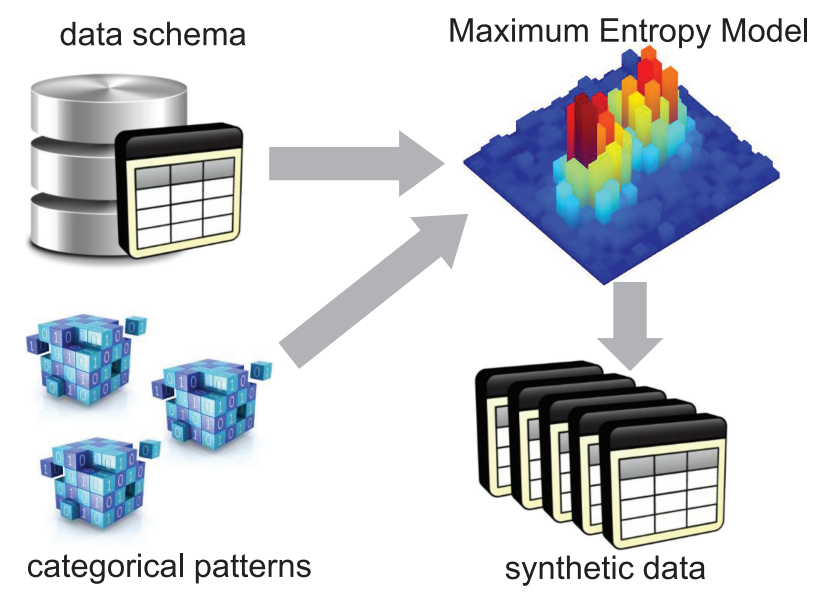

Fig. 1. Process of generating realistic synthetic data with our proposed approach.

(2) By exploring the structure of the categorical data space, we propose a partition scheme to make the maximum entropy model inference more efficient than the general case. We also present an efficient graph-based model inference algorithm.

(3) We propose a BIC-based heuristic to perform model selection wherein the simple and informative maximum entropy model will be chosen.

(4) Using results on both synthetic datasets and real US census data, we demonstrate that the proposed maximum entropy model is capable of recovering the underlying categorical data distribution and generating relevant synthetic populations.

\section{PRELIMINARIES}

Let $\mathcal{A}=\left\{A_{1}, A_{2}, \ldots, A_{q}\right\}$ denote a set of categorical random variables (or attributes), and $\mathcal{R}\left(A_{i}\right)=$ $\left\{a_{1}^{(i)}, a_{2}^{(i)}, \ldots, a_{k_{i}}^{(i)}\right\}$ represent the set of $k_{i}$ possible values for random variable $A_{i}$. Here, $|\cdot|$, e.g., $\left|\mathcal{R}\left(A_{i}\right)\right|$, is used to represent the cardinality of a set.

By a random categorical tuple, we mean a vector of categorical random variables, e.g., $T=$ $\left(A_{1}, A_{2}, \ldots, A_{q}\right)$, which is generated by some unknown probability distribution. The notation of $T\left(A_{i}\right)$ is used to represent the value of attribute $A_{i}$ in tuple $T$. The space of all the possible categorical tuples is denoted by $\mathcal{S}=\times_{i=1}^{q} \mathcal{R}\left(A_{i}\right)$, where $\times$. represents a series of Cartesian product over the given sets. Given a categorical pattern, which is defined as an ordered set $X=\left(A_{i} \mid A_{i} \in C, C \subseteq \mathcal{A}\right)$ over a subset of random variables $C \subseteq \mathcal{A}$, let $\mathcal{S}_{X}=\times_{A_{i} \in C} \mathcal{R}\left(A_{i}\right)$ represent the space that contains all the possible values of pattern $X$. An instantiation of pattern $X$ is defined as $x=\left(a_{j}^{(i)} \mid a_{j}^{(i)} \in \mathcal{R}\left(A_{i}\right), A_{i} \in C, C \subseteq \mathcal{A}\right)$, and $X\left(A_{i}\right)$ is used to represent the value of attribute $A_{i}$ in the pattern $X$.

For any pattern value $x$ associated with pattern $X$, we use the notation of $T=x$ if the corresponding random variables in $T$ equal to the values in $x$ and $p(T=x)$ to denote the probability of $T=\boldsymbol{x}$. Given a categorical dataset $D, \tilde{p}(T=\boldsymbol{x} \mid D)$ is used to denote the empirical probability of $T=\boldsymbol{x}$ in the dataset $D$. An indicator function $I_{X}(T=x): \mathcal{S} \rightarrow\{0,1\}$ of pattern $X$, which maps a categorical tuple to a binary value, is defined as follows:

$$
I_{X}(T=x)= \begin{cases}1, & \text { if } T=x \\ 0, & \text { otherwise }\end{cases}
$$


Table 1. Summary of Frequently Used Notation

\begin{tabular}{cl|cl}
\hline$A_{i}$ & Categorical random variable & $\mathcal{A}$ & A set of categorical random variable \\
$a^{(i)}$ & Value for random variable $A_{i}$ & $\mathcal{R}\left(A_{i}\right)$ & Set of possible values for $A_{i}$ \\
$|\cdot|$ & Set cardinality & $T$ & Random categorical tuple \\
$T\left(A_{i}\right)$ & Value of random variable $A_{i}$ in T & $X$ & Categorical pattern \\
$X\left(A_{i}\right)$ & Value of $A_{i}$ in pattern $X$ & $\boldsymbol{x}$ & Value instantiation of pattern $X$ \\
$\mathcal{S}$ & Entire categorical tuple space & $\mathcal{S}_{X}$ & Categorical tuple space spanned by \\
$p$ & Probability distribution & & pattern $X$ \\
$D$ & Categorical dataset & $\tilde{p}$ & Empirical probability distribution \\
$I_{X}(\cdot)$ & Indicator function of pattern $X$ & $H(p)$ & Entropy of probability distribution $p$ \\
& & $u, v$ & Maximum entropy model parameters \\
\hline
\end{tabular}

Given a probability distribution $p$ over the categorical tuple space $\mathcal{S}$, the entropy $H(p)$ with respect to $p$ is defined as follows:

$$
H(p)=-\sum_{T \in \mathcal{S}} p(T) \log p(T)
$$

The maximum entropy principle states that among a set of probability distributions $\mathcal{P}$ that comply with the given prior information about the data, the maximum entropy distribution

$$
p^{*}=\underset{p \in \mathcal{P}}{\operatorname{argmax}} H(p)
$$

will optimally use the current prior information and best summarize the data. Otherwise, it is fully unbiased. Table 1 summarizes the frequently used notations in this article.

Problem Statement. Given a set of categorical patterns $\mathcal{X}$ with associated empirical probabilities as the prior information of a dataset, find a probabilistic model $p$ that best utilizes the given prior information and helps regenerate categorical datasets that conform to the given prior information.

\section{CATEGORICAL MAXIMUM ENTROPY MODEL}

\subsection{Model Specification}

Suppose, we have a set of categorical patterns $\mathcal{X}=\left\{X_{i} \mid i=1,2, \ldots, n\right\}$ and an associated set of empirical probabilities $\tilde{P}=\left\{\tilde{p}\left(T=\boldsymbol{x}_{i, j} \mid D\right) \mid \boldsymbol{x}_{i, j} \in \mathcal{S}_{X_{i}}, i=1,2, \ldots, n\right\}$ as prior information about dataset $D$. Here, $\boldsymbol{x}_{i, j}$ denotes the $j$ th value of the pattern $X_{i}$. Notice that it is not necessary that every possible value of pattern $X_{i}$ in $\mathcal{S}_{X_{i}}$ is provided as part of the prior information here. Such prior information identifies a group of probability distributions $\mathcal{P}$ over $\mathcal{S}$ that agree with the empirical probabilities of the given categorical patterns. That is;

$$
\mathcal{P}=\left\{p \mid p\left(T=x_{i, j}\right)=\tilde{p}\left(T=x_{i, j} \mid D\right), \forall X_{i} \in \mathcal{X}, \tilde{p}\left(T=x_{i, j} \mid D\right) \in \tilde{P}\right\} .
$$

Following the maximum entropy principle [5], for all $p \in \mathcal{P}$, we are particularly interested in the maximum entropy distribution that optimally represents the given prior information. The famous theorem proved by Csiszár [5] (Theorem 3.1) shows that the maximum entropy distribution has an exponential form. In our categorical scenario, the maximum entropy distribution could be written as

$$
p^{*}(T)=u_{0} \prod_{X_{i} \in \mathcal{X}} \prod_{x_{i, j} \in \mathcal{S}_{X_{i}}}\left(u_{i, j}\right)^{I_{X_{i}}\left(T=\boldsymbol{x}_{i, j}\right)},
$$




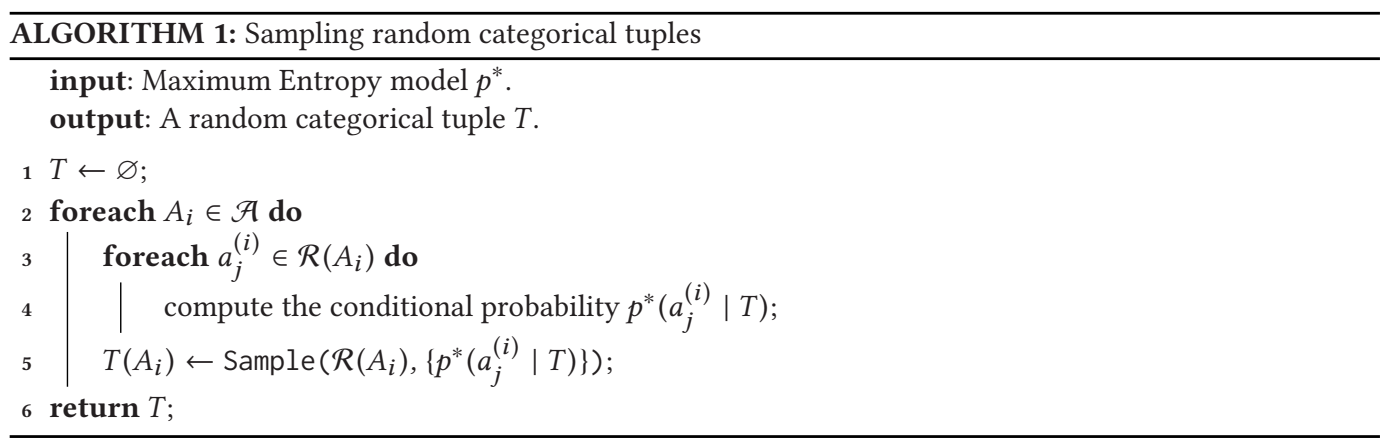

where $u_{i, j} \in \mathbb{R}$ are the model parameters associated with each model constraint specified in Equation (1), and $u_{0}$ is the normalizing constant.

\subsection{Incorporating Individual Attribute Frequencies}

The frequencies of individual attributes play an important role in the pattern analysis and discovery. Such frequencies characterize the attribute marginal distributions that convey basic information about the data currently under investigation, and yet are relatively easy to calculate from the data. Incorporating such individual attribute frequencies will enrich the categorical maximum entropy model, and make it more informative.

Although such individual attribute frequencies can be treated as part of the categorical pattern set $X$, considering the computation efficiency, which will be explained in detail in the next section, the categorical maximum entropy model treats them separately. Let $v_{i, j}$ denote the model parameters corresponding to the individual attribute constraints, then, the maximum entropy distribution can be factorized as follows:

$$
p^{*}(T)=u_{0} \prod_{X_{i} \in \mathcal{X}} \prod_{x_{i, j} \in \mathcal{S}_{X_{i}}}\left(u_{i, j}\right)^{I_{X_{i}}\left(T=x_{i, j}\right)} \times \prod_{A_{i} \in \mathcal{A}} \prod_{a_{j} \in \mathcal{R}\left(A_{i}\right)}\left(v_{i, j}\right)^{I_{A_{i}}\left(T=a_{j}\right)} .
$$

Notice that the second component involved with $v_{i, j}$ also follows the exponential form described in Equation (2). By introducing a normalizing constant $v_{0}$, an independent maximum entropy distribution $p_{\mathcal{A}}(T)$ that only involves individual attribute constraints could be defined as follows:

$$
p_{\mathcal{A}}(T)=v_{0} \prod_{A_{i} \in \mathcal{A}} \prod_{a_{j} \in \mathcal{R}\left(A_{i}\right)}\left(v_{i, j}\right)^{I_{A_{i}}\left(T=a_{j}\right)} .
$$

Combining Equations (3) and (4), the maximum entropy distribution that incorporates individual attribute frequencies would be specified as follows:

$$
p^{*}(T)=p_{\mathcal{A}}(T) \frac{u_{0}}{v_{0}} \prod_{X_{i} \in \mathcal{X}} \prod_{\boldsymbol{x}_{i, j} \in \mathcal{S}_{X_{i}}}\left(u_{i, j}\right)^{I_{X_{i}}\left(T=x_{i, j}\right)} .
$$

\subsection{Sampling Categorical Tuples from the Model}

The ideal way to sample categorical tuples from the model would be first computing the probabilities for each tuple $T \in \mathcal{S}$ under the maximum entropy distribution $p^{*}$, and then sampling the tuples from the complete tuple space $\mathcal{S}$ based on their probabilities. However, such straightforward approach is not feasible in practice when $\mathcal{S}$ is large enough.

Instead, we propose an attribute-wise sampling approach. Algorithm 1 summarizes the procedure that generates random categorical tuples from the maximum entropy model $p^{*}$. To sample 


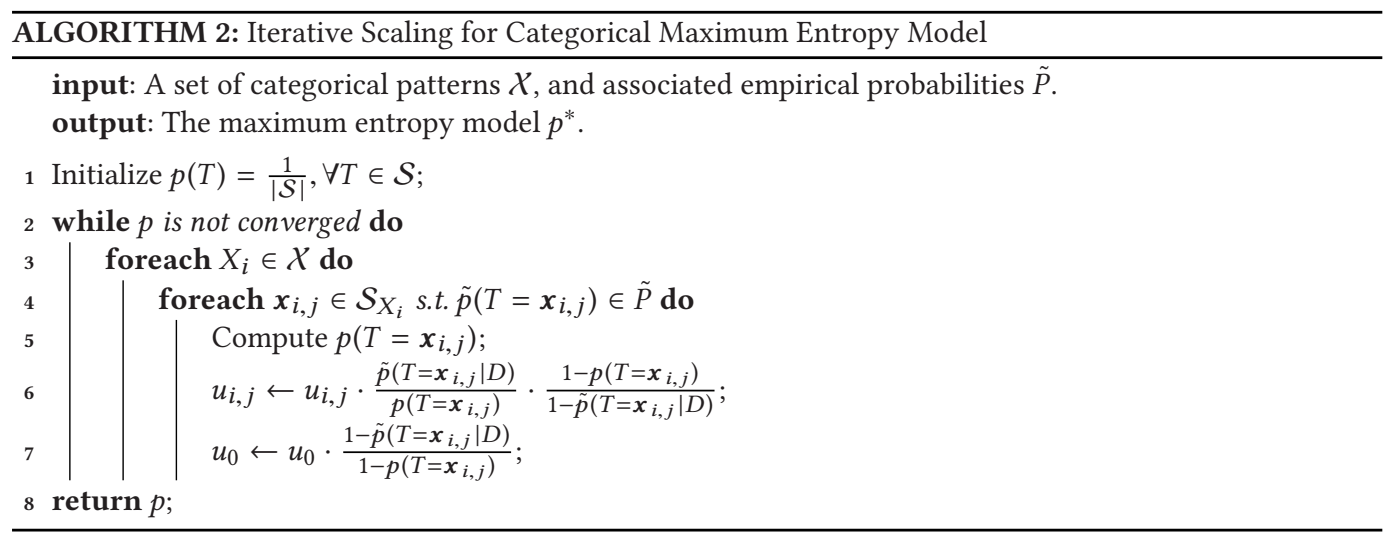

random tuples, we take the following steps. Starting with an empty categorical tuple $T$ (line 1), for each categorical attribute $A_{i} \in \mathcal{A}$, we compute the probability of each possible value $a_{j}^{(i)} \in \mathcal{R}\left(A_{i}\right)$ conditioned on those attribute values we have already sampled in tuple $T$ (line $3-4$ ). Based on the conditional probability distribution just calculated for attribute $A_{i}$, the value of $A_{i}$ in tuple $T$ is randomly sampled from its range $\mathcal{R}\left(A_{i}\right)$ (line 5 ). Notice that the order of selecting attributes could be random, and from statistical point of view, this should not affect the sampling result.

\section{MODEL INFERENCE}

In this section, we develop an efficient algorithm to infer the categorical maximum entropy model. To infer the categorical maximum entropy model, we need to find the values of model parameters $u_{0}$ and $u_{i, j}$ (also $v_{0}$ and $v_{i, j}$ if individual attribute constraints are involved). Our algorithm is built on the well-known Iterative Scaling [6] framework, which is described in Algorithm 2. The general idea of the algorithm is that starting from the uniform distribution, it iteratively updates each model parameter to make the distribution satisfy the corresponding constraint until it converges to the maximum entropy distribution. The proof of convergence for the Iterative Scaling algorithm is out of the scope for this article. Readers who are interested in the proof of convergence can refer to the paper by Darroch and Ratcliff [6] for details. A crucial step in the Iterative Scaling algorithm is to compute the probability of every categorical pattern $X_{i}=x_{i, j} \in \mathcal{X}$ under the current estimation of maximum entropy distribution $p$, which could be simply calculated as $p\left(T=\boldsymbol{x}_{i, j}\right)=\sum p(T)$ where $I_{X_{i}}\left(T=\boldsymbol{x}_{i, j}\right)=1$. However, such straightforward strategy is infeasible in our problem setting since it will result a computational complexity of $\prod_{A_{i} \in \mathcal{A}}\left|\mathcal{R}\left(A_{i}\right)\right|$ for a single model parameter update. In fact, querying maximum entropy models has been shown to be PP-hard [30]. To overcome such challenge, we present our proposed efficient model inference algorithm in the rest of this section.

\subsection{Efficient Model Inference}

In order to efficiently query the maximum entropy model during the iterative updates of the model parameters, we need to explore the particular structure of the tuple space $\mathcal{S}$ determined by the given pattern set $\mathcal{X}$. We will start with the simpler case where individual attribute constraints are not involved. After examining the exponential form of the maximum entropy distribution in Equation (2), we observe that for any two categorical tuples $T_{1}$ and $T_{2}$ in $\mathcal{S}$, if they contain the same subset of categorical patterns in $\mathcal{X}$, they will have the same probability under the maximum entropy distribution inferred based on $\mathcal{X}$. In other words, $\forall T_{1}, T_{2} \in \mathcal{S}$, if $I_{X_{i}}\left(T_{1}=\boldsymbol{x}_{i, j}\right)=I_{X_{i}}\left(T_{2}=\boldsymbol{x}_{i, j}\right)$ 
holds true for all $X_{i} \in \mathcal{X}$ and $\tilde{p}\left(T=\boldsymbol{x}_{i, j} \mid D\right) \in \tilde{P}$, then $p^{*}\left(T_{1}\right)=p^{*}\left(T_{2}\right)$. Based on such observation, we have the following definition of tuple block.

Definition 4.1. A tuple block $B$ is a set categorical tuples such that $\forall T_{1}, T_{2} \in B, I_{X_{i}}\left(T_{1}=\boldsymbol{x}_{i, j}\right)=$ $I_{X_{i}}\left(T_{2}=\boldsymbol{x}_{i, j}\right)$ holds true for all $X_{i} \in \mathcal{X}, \boldsymbol{x}_{i, j} \in \mathcal{S}_{X_{i}}$, and $\tilde{p}\left(T=\boldsymbol{x}_{i, j} \mid D\right) \in \tilde{P}$.

With the definition of tuple block, we could partition the entire categorical tuple space into several tuple blocks. When $|X| \ll|\mathcal{A}|$, the partition scheme introduced here could greatly reduce the dimensionality of the space we are working on. Here, we use $\mathcal{B}_{\mathcal{X}}$ to denote the tuple block space generated based on pattern set $\mathcal{X}$. Also, the definition of tuple block let us extend the indicator function defined over tuple space to the domain of tuple block, which is defined as follows:

$$
I_{X_{i}}\left(B \mid \boldsymbol{x}_{i, j}\right)=I_{X_{i}}\left(T=\boldsymbol{x}_{i, j}\right), \quad \forall X_{i} \in \mathcal{X}, T \in B .
$$

By introducing tuple blocks, we transfer the problem of computing categorical pattern probability $p\left(T=\boldsymbol{x}_{i, j}\right)$ on tuple space to the block space, which makes it possible to calculate $p\left(T=\boldsymbol{x}_{i, j}\right)$ in a reasonable time. In the context of tuple blocks, the pattern probability $p\left(T=x_{i, j}\right)$ would be

$$
p\left(T=x_{i, j}\right)=\sum_{\substack{B \in \mathcal{B}_{X}, I_{X_{i}}\left(B \mid x_{i, j}\right)=1}} p(B),
$$

where $p(B)$ is the probability for tuple block $B$. Since the probabilities for the categorical tuples within the same block are all the same, the probability for the tuple block $B$ is defined as follows:

$$
p(B)=\sum_{T \in B} p(T)=|B| \times u_{0} \prod_{X_{i} \in \mathcal{X}} \prod_{x_{i, j} \in \mathcal{S}_{X_{i}}}\left(u_{i, j}\right)^{I_{X_{i}}\left(B \mid x_{i, j}\right)} .
$$

Now, our problem comes down to how to organize the tuple block space $\mathcal{B}_{X}$ and efficiently compute the number of categorical tuples in each block, or in other words, the size $|B|$ of each tuple block $B$. In order to achieve that, we introduce a partial order on $\mathcal{B}_{X}$. Let

$$
\operatorname{attr}(B)=\bigcup_{\substack{X_{i} \in \mathcal{X}, I_{X_{i}}\left(B \mid \boldsymbol{x}_{i, j}\right)=1}} X_{i},
$$

which represents the set of attributes involved by the categorical patterns that tuple block $B$ contain. Then, we have the definition about the partial order over $\mathcal{B}_{\mathcal{X}}$ as described below.

Definition 4.2. Given any tuple blocks $B_{1}, B_{2} \in \mathcal{B}_{\mathcal{X}}, B_{1} \subseteq B_{2}$ if and only if the following conditions hold true:

(1) $\operatorname{attr}\left(B_{1}\right) \subseteq \operatorname{attr}\left(B_{2}\right)$;

(2) $B_{1}\left(A_{k}\right)=B_{2}\left(A_{k}\right), \forall A_{k} \in \operatorname{attr}\left(B_{1}\right) \cap \operatorname{attr}\left(B_{2}\right)$.

Here, $B\left(A_{k}\right)$ denotes the value of attribute $A_{k}$ in the tuple block $B$. It is easy to verify that Definition 4.2 satisfies the property of reflexivity, antisymmetry, and transitivity.

With the partial order $\subseteq$ defined on $\mathcal{B}_{X}$ here, it is natural to organize the tuple blocks into a hierarchical graph structure. That is, if tuple block $B_{k} \subseteq B_{l}$, block $B_{l}$ is organized as the child of block $B_{k}$. Algorithm 3 illustrates how such block graph is constructed and maintained. The algorithm starts with the graph that has only one block represented by $\varnothing$ indicating that none of the categorical patterns is involved in this block (line 1). We will refer this block as root block in the rest of this section. Then, for each of the categorical pattern $X_{i} \in \mathcal{X}$ and its possible value $\boldsymbol{x}_{i, j}$, we attempt to create a new tuple block by merging it with every existing block $B_{k}$ from root level to leaf level (without child blocks) in the current block graph $G$ if they are compatible (line 4). 


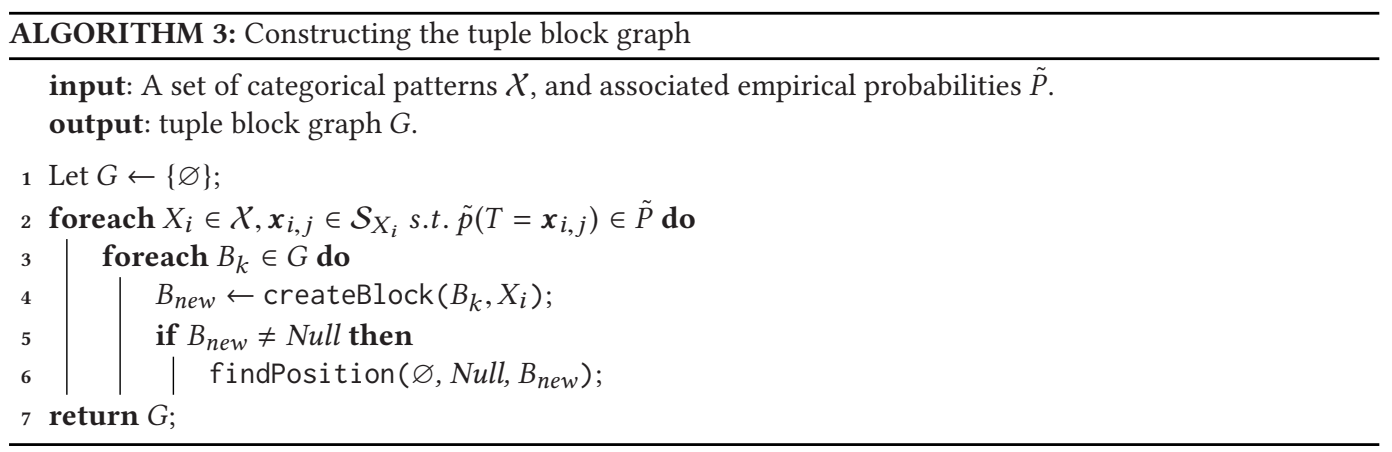

A categorical pattern $X_{i}$ is not compatible with tuple block $B_{k}$ if $\operatorname{attr}\left(B_{k}\right) \cap X_{i} \neq \emptyset$, and $\exists A_{i} \in$ $\operatorname{attr}\left(B_{k}\right) \cap X_{i}$ such that $B_{k}\left(A_{i}\right) \neq X_{i}\left(A_{i}\right)$. If a new tuple block $B_{\text {new }}$ is created, it is obvious that for all $X_{l} \in \mathcal{X}, I_{X_{l}}\left(B_{k} \mid \boldsymbol{x}_{l, j}\right)=1$, we have $I_{X_{l}}\left(B_{\text {new }} \mid \boldsymbol{x}_{l, j}\right)=1$ and also $I_{X_{i}}\left(B_{\text {new }} \mid \boldsymbol{x}_{i, j}\right)=1$. Finally, the new tuple block $B_{\text {new }}$ will be added into the current block graph $G$ based on the partial order described in Definition 4.2 (line 6).

To be more specific, Algorithm 4 illustrates how the procedure findPosition inserts a new tuple block into the block graph $G$ in a recursive manner. Depending on the relationship between the current block $B_{\text {curr }}$ we are visiting and the new block $B_{\text {new }}$, the insertion operation could be classified into four scenarios.

Case 1. $B_{\text {new }}$ and $B_{\text {curr }}$ are the same tuple block. Two tuple block $B_{k}$ and $B_{l}$ are considered to be the same if they cover the same set of categorical patterns, e.g., $\forall X_{i} \in \mathcal{X}, \boldsymbol{x}_{i, j} \in \mathcal{S}_{X_{i}}$ s.t. $\tilde{p}\left(T=\boldsymbol{x}_{i, j}\right) \in \tilde{P}$, we have $I_{X_{i}}\left(B_{k} \mid \boldsymbol{x}_{i, j}\right)=I_{X_{i}}\left(B_{l} \mid \boldsymbol{x}_{i, j}\right)$. Since block $B_{\text {new }}$ and $B_{\text {curr }}$ are the same and $B_{\text {curr }}$ is already part of the block graph, inserting $B_{\text {new }}$ into block graph is not necessary any more. Thus, we simply return Success in this scenario (line 1-2).

Case 2. $B_{\text {new }} \subseteq B_{\text {curr }}$. In this case, the new tuple block $B_{\text {new }}$ should be inserted between block $B_{\text {last }}$ and $B_{\text {curr }}$, where $B_{\text {last }}$ is the last visited tuple block. To achieve this, block $B_{\text {curr }}$ is first removed from the child block set of $B_{\text {last }}$, and added as a child block of $B_{\text {new }}$. Finally, the new block $B_{\text {new }}$ is inserted as a child block of $B_{\text {last }}$, and Success is returned (line 3-7).

Case 3. $B_{\text {curr }} \subseteq B_{\text {new }}$. In this scenario, the new tuple block $B_{\text {new }}$ should be inserted as a descendant of the current block $B_{\text {curr }}$. Depending on whether the block $B_{\text {curr }}$ has any child blocks, the insertion operation can be further divided into two sub-cases:

- Case 3.1: block $B_{\text {curr }}$ has no child block. In this scenario, the new block $B_{\text {new }}$ is directly inserted as a new child of $B_{\text {curr }}$ (line 9-11);

- Case 3.2: block $B_{\text {curr }}$ has child blocks. Then, for each child block of $B_{\text {curr }}$, the findPosition procedure is recursively performed to find the correct position to insert block $B_{\text {new }}$ (line 19-24). If none of these operations succeeds, block $B_{\text {new }}$ will be inserted as a new child block of $B_{\text {curr }}$ (line 25-26). At last, the descendants of the child blocks of $B_{\text {curr }}$ on which the findPosition procedure failed to insert the block $B_{\text {new }}$ are further examined to see whether any of them could satisfy the partial order with block $B_{\text {new }}$ and be added as a child block of $B_{\text {new }}$ (line 14 , checkDescendant procedure).

Case 4. $B_{\text {new }}$ does not have any particular relationship with $B_{\text {curr }}$. In this case, nothing needs to done with the tuple blocks $B_{\text {curr }}$ and $B_{\text {new }}$, and Fail is simply returned to indicate that the attempt to insert block $B_{\text {new }}$ is failed. 


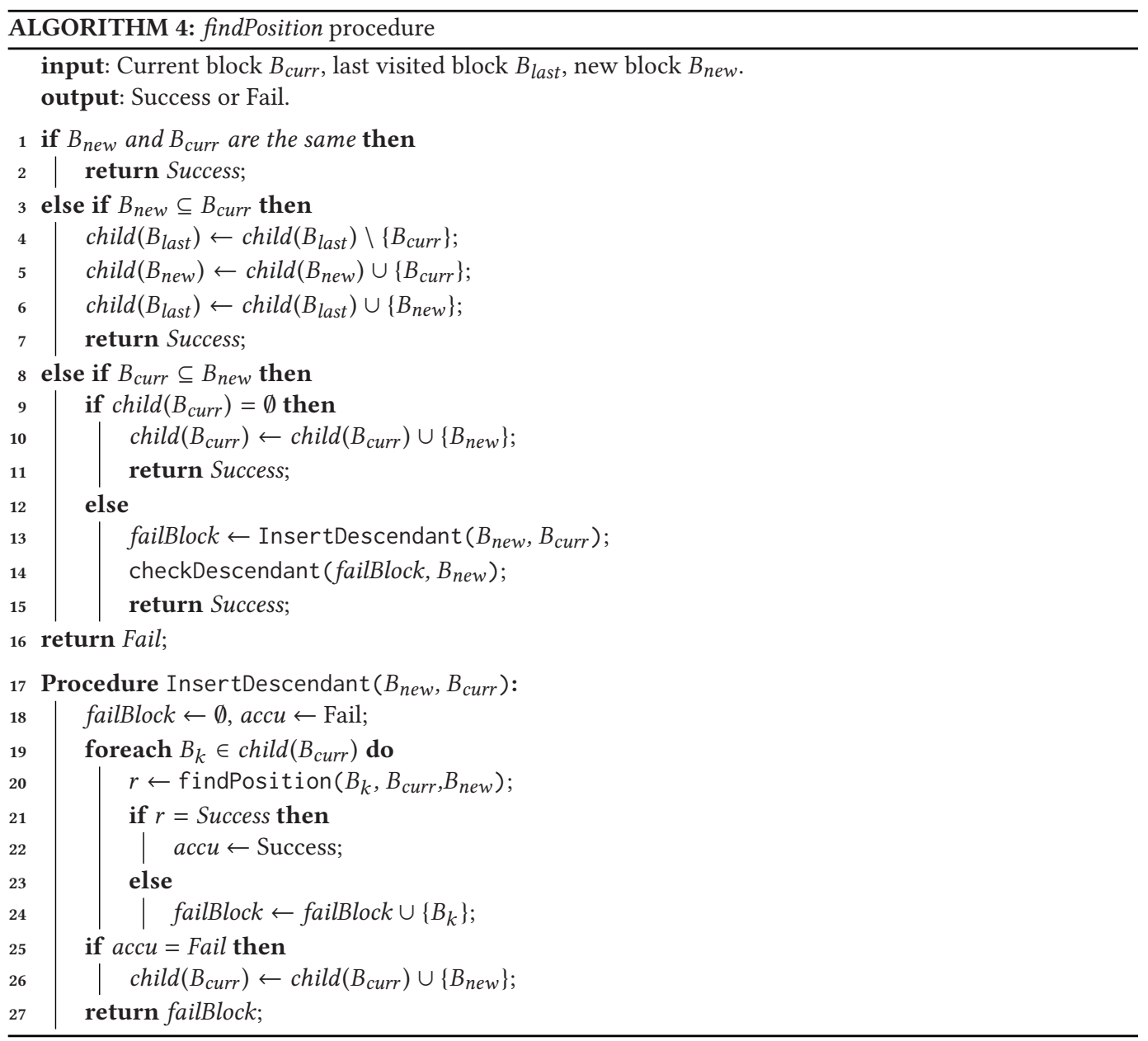

Figure 2 shows an example of constructing such hierarchical block graph on a small toy dataset with six attributes and three categorical patterns. With the block graph $G$, the size of the tuple block could be easily calculated using the set inclusion-exclusion principle. We first define the cumulative size of a tuple block $B$, which is given by

$$
\operatorname{cum}(B)=\prod_{A_{i} \in \mathcal{A} \backslash \operatorname{attr}(B)}\left|\mathcal{R}\left(A_{i}\right)\right| .
$$

Then, the actual block size for block $B$ could be computed as

$$
|B|=\operatorname{cum}(B)-\sum_{B_{k} \in \mathcal{B}_{X}, B \subseteq B_{k}}\left|B_{k}\right| .
$$

In the block graph $G$, the tuple blocks that satisfy $B_{k} \in \mathcal{B}_{\mathcal{X}}, B \subseteq B_{k}$ are simply those descendant blocks of $B$. Algorithm 5 describes the procedure of computing block size for each tuple block in $\mathcal{B}_{X}$ with the block graph $G$, where $\operatorname{desc}(B)$ represents the set of descendant blocks of $B$ in the graph G. 


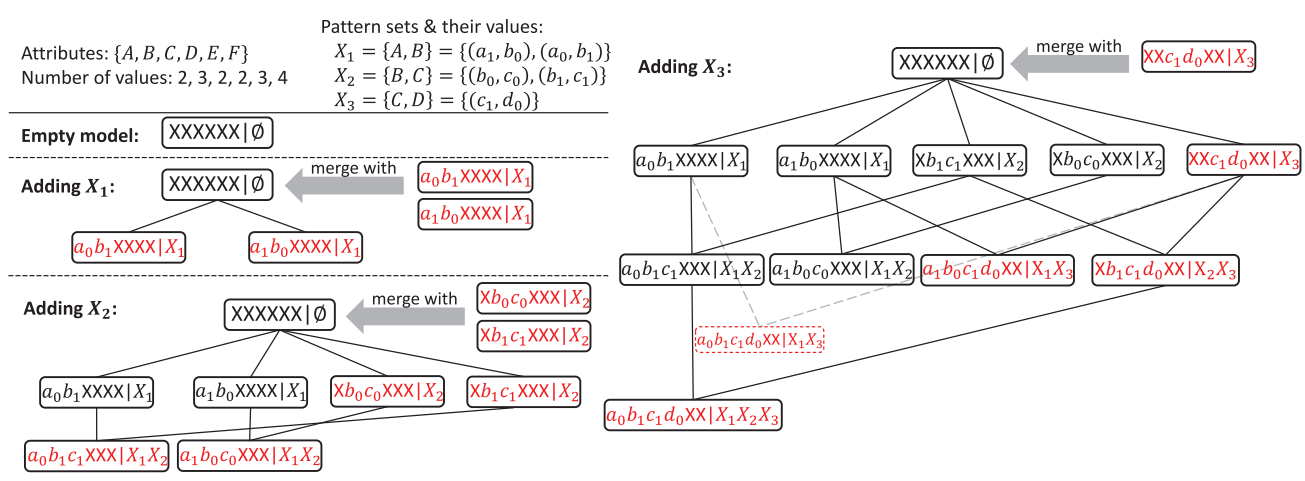

Fig. 2. Example of constructing the tuple block graph on a toy dataset with six attributes and three categorical patterns. The blocks marked with red denote the new tuple blocks created in each iteration by adding new categorical patterns.

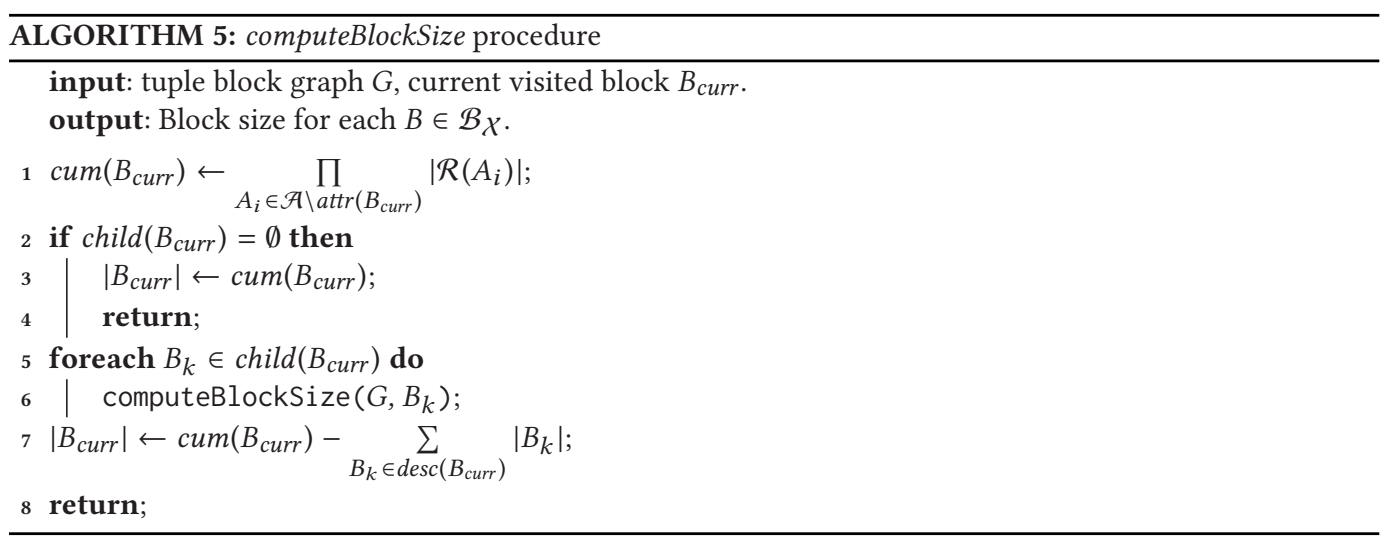

When individual attribute constraints are taken into account, the problem becomes a little more complicated. However, it is obviously not feasible to combine the individual attribute constraints with the categorical pattern constraints together and construct the tuple block graph. This will make the tuple block space blow up. Instead, as we mentioned previously in Section 3, the individual attribute constraints are modeled with a separate maximum entropy distribution $p_{\mathcal{A}}$, defined in Equation (4), which only considers these constraints. The block graph $G$ is still constructed based on the categorical patterns in $\mathcal{X}$, which will exactly have the same structure as before. In this case, following the same logic, the probability for tuple block $B$ becomes

$$
p(B)=p_{\mathcal{A}}(B) \cdot \frac{u_{0}}{v_{0}} \cdot \prod_{X_{i} \in \mathcal{X}} \prod_{\boldsymbol{x}_{i, j} \in \mathcal{S}_{X_{i}}}\left(u_{i, j}\right)^{I_{X_{i}}\left(B \mid x_{i, j}\right)},
$$

where $p_{\mathcal{A}}(B)=\sum_{T \in B} p_{\mathcal{A}}(T)$ denotes the probability of tuple block $B$ under the separate maximum entropy distribution $p_{\mathcal{A}}$. Thus, the problem of computing the probability $p\left(T=\boldsymbol{x}_{i, j}\right)$ becomes calculating probabilities of tuple blocks $p_{\mathcal{A}}(B)$ for each $B \in \mathcal{B}_{\mathcal{X}}$. Since $p_{\mathcal{A}}$ only takes the individual attribute constraints into account, every attribute is independent of each other under the maximum entropy distribution $p_{\mathcal{A}}$. Similar to the cumulative size of a tuple block, we define the cumulative 
probability of a tuple block under $p_{\mathcal{A}}$ as

$$
p_{\mathcal{A}}^{(c)}(B)=\prod_{A_{i} \in \operatorname{attr}(B)} p_{\mathcal{A}}\left(T=a_{j}^{(i)}\right),
$$

where $a_{j}^{(i)}$ is the value of attribute $A_{i}$ associated with tuple block $B$. With the exponential form described in Equation (4), it is not difficult to verify that the probability of $T=a_{j}^{(i)}$ under maximum entropy distribution $p_{\mathcal{A}}$ is:

$$
p_{\mathcal{A}}\left(T=a_{j}^{(i)}\right)=\frac{v_{i, j}}{\sum_{l=1}^{k_{i}} v_{i, l}} .
$$

Again, to compute $p_{\mathcal{A}}(B)$ for all $B \in \mathcal{B}_{\mathcal{X}}$ with the set inclusion-exclusion principle, we could directly apply the computeBlockSize procedure with $|B|$ and $\operatorname{cum}(B) \operatorname{replaced}$ by $p_{\mathcal{A}}(B)$ and $p_{\mathcal{A}}^{(c)}(B)$ respectively.

Notice that the model parameters $v_{i, j}$ also need to be updated in the Iterative Scaling framework. However, the block graph $G$ is constructed without considering individual attribute patterns, which makes it difficult to compute the probabilities of these individual attribute patterns under the maximum entropy model directly from the block graph $G$. In order to get these probabilities, we treat these individual attribute patterns as arbitrary categorical patterns and query their probabilities from the maximum entropy model. The detail of querying the maximum entropy model will be described in the next section.

Finally, the model inference algorithm could be further optimized in the following way. Suppose the categorical patterns in $X$ could be divided into two disjoint groups, e.g., $X_{1}, X_{2} \subset \mathcal{X}$ and $X_{1} \cup$ $X_{2}=X$ such that $\forall X_{1} \in \mathcal{X}_{1}, \forall X_{2} \in \mathcal{X}_{2}$ we have $X_{1} \cap X_{2}=\emptyset$. In this case, the maximum entropy model $p_{X}^{*}$ over $X$ could be factorized into two independent components $p_{X_{1}}^{*}$ and $p_{X_{2}}^{*}$ such that $p_{\mathcal{X}}^{*}=p_{\mathcal{X}_{1}}^{*} \cdot p_{\mathcal{X}_{2}}^{*}$. Furthermore, $p_{\mathcal{X}_{1}}^{*}$ and $p_{\mathcal{X}_{2}}^{*}$ only rely on pattern set $\mathcal{X}_{1}$ and $\mathcal{X}_{2}$, respectively. Such decomposition greatly reduces the sizes of tuple block spaces $\mathcal{B}_{X_{1}}$ and $\mathcal{B}_{X_{2}}$ compared to the original $\mathcal{B}_{X}$, and could also be extended to the scenario when there are multiple such disjoint pattern groups. Due to the independence between these maximum entropy components, they can also be inferred parallelly to further speed up the model inference process.

\subsection{Querying the Model}

Given an arbitrary categorical pattern $X^{\prime} \notin X$ with associated value $\boldsymbol{x}^{\prime}$, to query its probability under the maximum entropy distribution $p^{*}$, we perform the following operations. Let $\mathcal{X}^{\prime}=\mathcal{X} \cup$ $\left\{X^{\prime}\right\}$, and a temporary tuple block graph $G^{\prime}$ is constructed by applying the procedure described in Algorithm 3 over categorical pattern set $X^{\prime}$. Then, the size of each tuple block in graph $G^{\prime}$ is computed by calling computeBlockSize procedure, and the probability of categorical pattern $X^{\prime}$ is given by

$$
p^{*}\left(T=x^{\prime}\right)=\sum_{\substack{B \in \mathcal{B}_{X^{\prime}} \\ I_{X^{\prime}}\left(B \mid \boldsymbol{x}^{\prime}\right)=1}} p^{*}(B) .
$$

\subsection{Computational Complexity}

Constructing the tuple block graph (Algorithm 3) requires $\left|\mathcal{B}_{X}\right|$ insertion operations. Since the block graph has a hierarchical structure, let's define the depth of the tuple block graph as the maximum number of hops (parent-child relationship) from the root block $\varnothing$ to the leaf block. Notice that the parent-child relationship between tuple blocks in the graph is based on the partial order described in Definition 4.2, which indicates that the maximum possible depth of the tuple block graph would be $|\mathcal{A}|$. Thus, the complexity of constructing the tuple block graph in the worst 
case would be $O\left(|\mathcal{A}| \cdot\left|\mathcal{B}_{X}\right|\right)$. When inferring the maximum entropy model, the probability of each tuple block needs to be calculated for each model parameter update (Algorithm 5), which results a complexity of $O\left(\left|\mathcal{B}_{X}\right|\right)$. If we let $N$ denote the number of model parameters, the complexity of inferring the maximum entropy model would be $O\left(K \cdot N \cdot\left|\mathcal{B}_{\chi}\right|\right)$, where $K$ is the number of iterations required for the proposed inferring algorithm to converge.

\section{MODEL SELECTION}

In order to discover the most informative prior information from the pattern set $\mathcal{X}$, we adopt the BIC, defined as:

$$
B I C_{X}=-2 \log \mathcal{L}_{X}+N \cdot \log |D|,
$$

where $\log \mathcal{L}_{X}$ denotes the log-likelihood of the maximum entropy model inferred over the pattern set $\mathcal{X}, N$ represents the number of model parameters, and $|D|$ is the number categorical tuples in the dataset $D$. With the exponential form of the maximum entropy distribution specified in Equation (2), its log-likelihood given dataset $D$ is equal to

$$
\log \mathcal{L}_{\mathcal{X}}=\sum_{T \in D} \log p^{*}(T)=|D|\left(\log u_{0}+\sum_{X_{i} \in \mathcal{X}} \sum_{\boldsymbol{x}_{i, j} \in \mathcal{S}_{X_{i}}} \tilde{p}\left(T=\boldsymbol{x}_{i, j} \mid D\right) \cdot \log u_{i, j}\right) .
$$

The ideal approach to select the most informative categorical patterns from the pattern set $\mathcal{X}$ would be finding a subset of $\mathcal{X}$ that minimizes the BIC score of the model. However, notice that this approach involves a number of model inference operations which is proportional to the number of subsets of $\mathcal{X}$. Considering the computation required for the model inference, this method may be infeasible in practice. Hence, we resort to heuristics. Basically, what we desire are the patterns whose empirical probabilities diverge most from their probabilities under current maximum entropy model. In this case, they will contain the most new information compared to what the model already knows. Thus, we borrow the idea from Kullback-Leibler (KL) divergence, where we make the probability of the categorical pattern $X$ under consideration as one term and the rest of the probability mass as the other term. To be more specific, the heuristic we use is defined as

$$
h(\alpha, \beta)=\alpha \log \frac{\alpha}{\beta}+(1-\alpha) \log \frac{1-\alpha}{1-\beta} .
$$

Instead of directly searching in the space of power set of $\mathcal{X}$, we adopt an iterative search strategy. Starting from the empty model without any prior information, in each iteration, we choose the pattern $X \in \mathcal{X}$ that maximizes the heuristic $h\left(p^{*}(T=x), \tilde{p}(T=x \mid D)\right)$ to update the current maximum entropy model. Here, $p^{*}(T=x)$ and $\tilde{p}(T=x \mid D)$ denote the probability of pattern $X$ under current maximum entropy model and its empirical probability in the given dataset $D$, respectively. As the model incorporates more and more patterns in $\mathcal{X}$, it becomes more certain about the data, and the negative log-likelihood decreases. However, the model becomes more complicated at the same time, and the penalty term in BIC becomes large. This procedure continues until the BIC score does not decrease any more.

\section{EXPERIMENTAL RESULTS}

\subsection{Synthetic Data Generation}

To evaluate the proposed maximum entropy model against the true generating distribution of categorical data, we generate synthetic datasets. Usually when the entire categorical data space is large, it is infeasible to specify an exact generating distribution for categorical data. Thus, we generate the synthetic data $D$ with the following approach. 


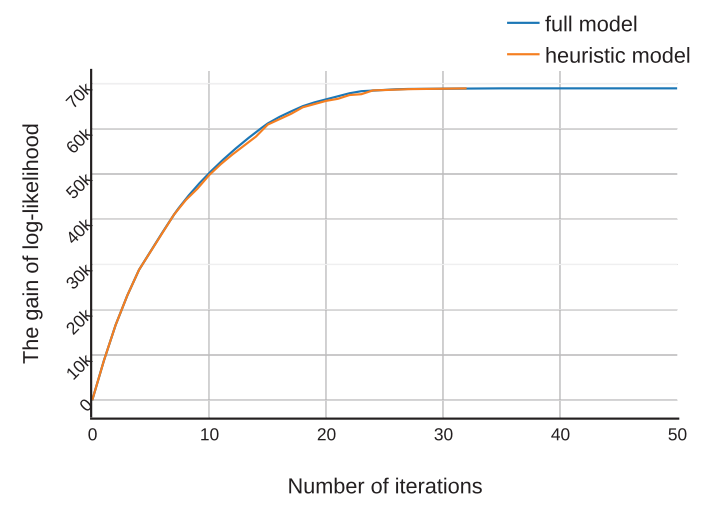

Fig. 3. The gain of the log-likelihood of the full model and heuristic model compared to the base line model. The blue line and orange line are so close that they overlap with each other in some iterations. Also, notice that orange line for heuristic model stops early due to the model selection with BIC.

A set of categorical attributes $\mathcal{A}$ is first generated, and the number of possible values for each attribute $A_{i} \in \mathcal{A}$ is randomly sampled from a given range. Each categorical attribute $A_{i}$ is associated with a random generated probability distribution (marginal distribution) that specifies the probability of each possible value of $A_{i}$. In order to enforce dependencies between attributes, a set of categorical patterns $\mathcal{X}$ is generated and each of these patterns is associated with a probability. To generate a categorical tuple in the synthetic dataset, we sample from a Bernoulli distribution parameterized by the pattern frequency of each $X \in \mathcal{X}$ to determine whether this tuple should contain this pattern or not. If conflicts occur, the current pattern $X$ will not be added into the tuple. For the rest of the attributes that are not covered by any of these patterns in $\mathcal{X}$, their values in the generated categorical tuple are sampled independently from their corresponding marginal distributions respectively. Such process is repeated to obtain the desired number of categorical tuples in the synthetic dataset. In our experiments, we set $|\mathcal{A}|=100,|X|=50$, and $|D|=10,000$. All the experiments were conducted on a 80 -core Xeon $2.4 \mathrm{GHz}$ machine with $1 \mathrm{~TB}$ memory, and the results were averaged across 40 independent runs.

\subsection{Results on Synthetic Data}

We first verify that the heuristic function $h(\alpha, \beta)$ proposed in Section 5 could discover the most informative patterns from $\mathcal{X}$ based on the current knowledge that the model already knows. We refer the maximum entropy model inferred with entire pattern set $\mathcal{X}$ and all the individual attribute frequencies as full model, and the maximum entropy model selected by the heuristic and BIC as heuristic model. Notice that in the heuristic model, individual attribute frequencies are also taken into account. In this experiment, we iteratively updated the model with the patterns in $\mathcal{X}$, and measured the log-likelihood in each iteration. However, using BIC to select the model may result different number of patterns incorporated over different synthetic datasets. Thus, we report the results over a single synthetic dataset here. For the full model, the pattern in $X$ that maximized the log-likelihood in each iteration was selected and added to the model.

Figure 3 illustrates the gain of the log-likelihood as the model incorporates more and more patterns in $\mathcal{X}$. As expected, the gain of the log-likelihood of the full model is larger in some iterations since it identifies the optimal pattern in each iteration with respect to the likelihood. We also observe that although not optimal, the log-likelihood of the heuristic model approximates that of the full model quite well, which demonstrates that the proposed heuristic successfully identifies the 
Table 2. Comparison of the Approximate KL-divergence Measure Between Full Model, Heuristic Model and Baseline Model.

Standard Errors are Shown in the Parentheses

\begin{tabular}{ccccc}
\hline & & Full model & Heuristic & Baseline \\
\hline \multirow{4}{*}{ All } & $\hat{K L}\left(p^{*}, p^{\prime}\right)$ & $\mathbf{0 . 0 0 6 0 1 3}$ & 0.009344 & 1.8126 \\
& & $\mathbf{0 . 0 1 9 8 3})$ & $(0.02516)$ & $(0.3534)$ \\
& $\hat{K L}\left(\tilde{p}, p^{\prime}\right)$ & $\mathbf{0 . 1 7 6 7}$ & 0.1840 & 1.9823 \\
& & $\mathbf{0 . 0 2 4 0 4})$ & $(0.02805)$ & $(0.3600)$ \\
\hline \multirow{4}{*}{ Multi-attribute } & $\hat{K} L\left(p^{*}, p^{\prime}\right)$ & $\mathbf{0 . 0 0 5 8 7 1}$ & 0.009204 & 1.8126 \\
& & $\mathbf{0 . 0 1 9 7 4 )}$ & $(0.02501)$ & $(0.3534)$ \\
& $\hat{K L}\left(\tilde{p}, p^{\prime}\right)$ & $\mathbf{0 . 0 2 8 3 7}$ & 0.03322 & 1.8330 \\
& & $\mathbf{0 . 0 1 6 8 5})$ & $(0.02339)$ & $(0.3579)$ \\
\hline
\end{tabular}

Note: The boldface values indicate these values are better than the rest of values in the table.

relatively informative patterns in each iteration. In the last few iterations, the gain of log-likelihood of the full model barely changes. This indicates that the patterns selected in these iterations are less informative or even redundant.

To assess the quality of the reconstruction, we aim to apply the KL divergence measure. However, in practice, it is very difficult to compute the KL divergence between the entire maximum entropy distribution and data generating distribution for the categorical data due to the large categorical tuple space. As a tradeoff, we use the probabilities of patterns in pattern set $\mathcal{Y}$ to characterize the probability distributions for categorical data in both scenarios, and define the following approximate KL-divergence measure:

$$
\hat{K L}\left(p^{*}, p^{\prime}\right)=\sum_{X \in \mathcal{Y}}\left[p^{*}(X) \log \frac{p^{*}(X)}{p^{\prime}(X)}+\left(1-p^{*}(X)\right) \log \frac{1-p^{*}(X)}{1-p^{\prime}(X)}\right] .
$$

Here, $p^{*}$ and $p^{\prime}$ denote the maximum entropy distribution and data generating distribution respectively, and pattern set $\mathcal{Y}$ could be only categorical pattern set $\mathcal{X}$ or $\mathcal{X} \cup \mathcal{A}$ if individual attribute frequencies are considered. We also compute the $\hat{K L}\left(\tilde{p}, p^{\prime}\right)$ to compare the empirical probability distribution, say $\tilde{p}$, in the samples generated by the categorical maximum entropy model with the true data generating distribution. In this experiment, we computed $\hat{K} L\left(p^{*}, p^{\prime}\right)$ and $\hat{K L}\left(\tilde{p}, p^{\prime}\right)$ for both full model and heuristic model. For comparison purpose, we used independent attribute model $p_{\mathcal{A}}$ where each categorical attribute is independent of each other as the baseline model. For each of these models under consideration, 1,000 categorical data samples were generated to compute empirical probability distribution $\tilde{p}$.

Table 2 compares these approximate KL-divergence measures for the scenarios where $\boldsymbol{Y}=$ $\mathcal{X} \cup \mathcal{A}$ (row All in Table 2) and $\mathcal{Y}=\mathcal{X}$ (row Multi-attribute in Table 2). In Table 2, the small approximate KL-divergence values for the full model and the heuristic model in the row All indicate that the categorical maximum entropy distributions converge to the underlying data generation distribution, and the samples generated by these two models successfully maintain the properties of the data generation distribution. More important, the small approximate KL-divergence values in the row Multi-attribute of Table 2 also indicate that the inferred categorical maximum entropy models successfully capture the various multivariate dependencies among multiple categorical attributes. All these results demonstrate that our model is capable of recovering the true categorical data distribution and maintaining its dependency structures. When compared to the baseline model, our model outperforms several magnitudes in term of estimation accuracy. 
Table 3. Comparison of Model Preparation Time $\left(t_{\text {pre }}\right)$, Model Inference Time $\left(t_{\text {infer }}\right)$ and Data Sampling Time $\left(t_{\text {sample }}\right)$ Between Full Model and Heuristic Model (In Seconds). Standard

Errors are Shown in the Parentheses

\begin{tabular}{cccc}
\hline & $t_{\text {pre }}$ & $t_{\text {infer }}$ & $t_{\text {sample }}$ \\
\hline \multirow{2}{*}{ Full model } & 2750.432 & 22.553 & 1.828 \\
& $(1512.416)$ & $(14.341)$ & $(1.002)$ \\
Heuristic model & $\mathbf{1 5 . 1 0 3}$ & $\mathbf{1 1 . 1 5 0}$ & $\mathbf{0 . 4 8 8}$ \\
& $(7.844)$ & $(6.549)$ & $(0.234)$ \\
\hline
\end{tabular}

Note: The boldface values indicate these values are better than the rest of values in the table.

We also measure the time required to prepare the pattern set that serves as prior information of the model $t_{\text {pre }}$, the time to infer the maximum entropy model $t_{\text {infer }}$, and the time to sample a single categorical tuple from the model $t_{\text {sample }}$. Here, for the full model, $t_{\text {pre }}$ refers to the time required to arrange the pattern set $\mathcal{X}$ into the same order used in the iterative model update procedure in the first experiment, where the categorical pattern that maximizes the log-likelihood is chosen in each iteration. Table 3 compares the runtime performance between the full model and the heuristic model. With the informative as well as simple model selected by the heuristic function $h(\alpha, \beta)$ and BIC, the heuristic model requires much less time to infer the maximum entropy distribution and sample categorical tuples from the model.

\subsection{Results on Real Data}

To evaluate the performance of the proposed categorical maximum entropy model on real data, we study the problem of generating synthetic populations with US census data. Specifically, we use the 2012 American Community Survey (ACS) 1-year summary data [33], which contains aggregated statistics about age, sex, race, income, and many other features. Some of these features, e.g., sex and race, are perfect categorical attributes for the proposed maximum entropy model. Although some other features, e.g., age and income, are numerical, they are binned into several ranges based on their values, and treated here as categorical attributes.

In our experiments, we chose the state of Virginia as our study case. Among all the features in the ACS summary data, we selected sex, age, race, income, occupation, marital status, means of transportation to work, education level, and health insurance coverage as the set of categorical attributes. We converted the corresponding aggregated statistics in the ACS summary data into categorical patterns, and inferred the heuristic model over these patterns. Figure 4 describes the gain of the log-likelihood of the heuristic model, and the approximate KL-divergence measure between the inferred maximum entropy distribution and the empirical data distribution in the Virginia ACS summary data is $\mathbf{0 . 0 0 0 1 9 7 5}$. Notice that in Figure 4, the last data point marked with a cross indicates the gain of the log-likelihood of the full model where all the categorical patterns in the Virginia ACS summary data are taken into account. As we can see from the figure, the gain of the log-likelihood of the final heuristic model is quite close to that of the full model, which indicates that the heuristic model discovers and incorporates the majority of the knowledge in the Virginia ACS summary data. Combined with the small value of the approximate KL-divergence measure, these results demonstrate that the proposed categorical maximum entropy model is able to well estimate the categorical data distribution from real data. Table 4 shows the most informative patterns selected by the proposed heuristic. 


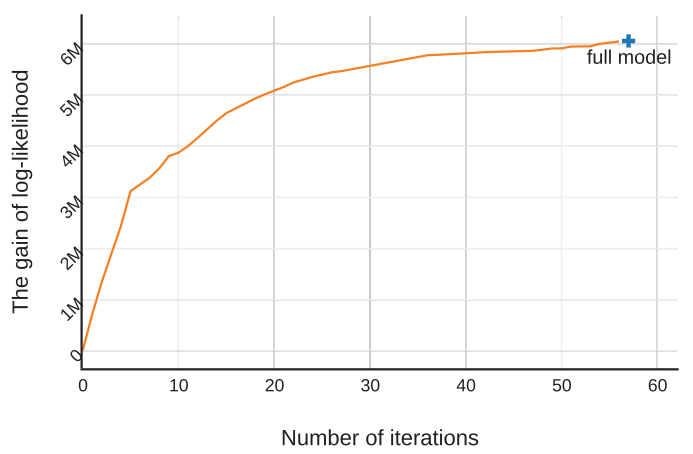

Fig. 4. The gain of the negative log-likelihood of the model compared to the baseline model (model at iteration 0 ) over the Virginia ACS summary data. The data point marked with a cross denotes the negative log-likelihood of the full model, where all the categorical patterns in the Virginia ACS summary data are considered.

Table 4. Top Categorical Patterns Selected by the Heuristic Model from the Virginia ACS Summary Data

\begin{tabular}{ccc}
\hline Patterns & Number of possible values & Number of selected values \\
\hline \{means of transportation & 49 & 34 \\
to work, occupation\} & 8 & 2 \\
\{sex, income & 10 & 2 \\
$\{$ sex, marital status\} & 8 & 1 \\
\{sex, age & & \\
\hline
\end{tabular}


Fig. 5. Comparison of single attribute marginal distributions between the true statistics in Virginia ACS summary data and samples generated by the categorical maximum entropy model for the attributes Sex and Income.

We also sampled 3,000 synthetic individuals with the inferred heuristic model for Virginia, and calculated the empirical marginal distributions for all of the individual attributes and all of the multi-attribute categorical patterns that appear in the Virginia ACS summary data. Notice that for attributes Marital status, Means of transportation to work, Occupation, and Education level, the population considered in the ACS summary data is not the entire population of Virginia state. Thus, we add an additional value for these attributes, e.g., the value Others under 15 years old for the attribute Marital status, to denote the proportion of the entire population that is not taken into account in the ACS summary data. Figures 5-8 show single-attribute and multi-attribute marginal 


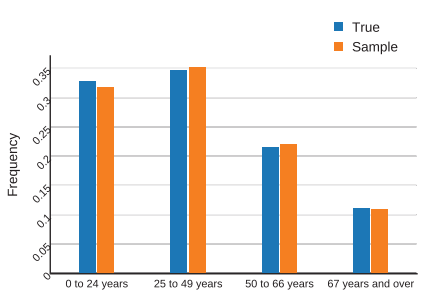

Age
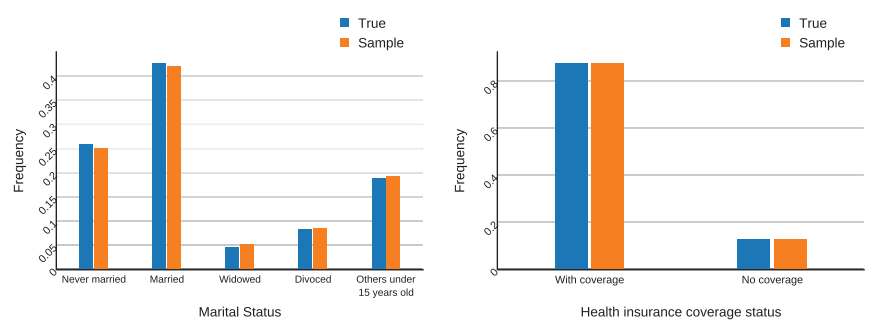

Fig. 6. Comparison of single attribute marginal distributions between the true statistics in Virginia ACS summary data and samples generated by the categorical maximum entropy model for the attributes Age, Marital status, and Health insurance coverage status.
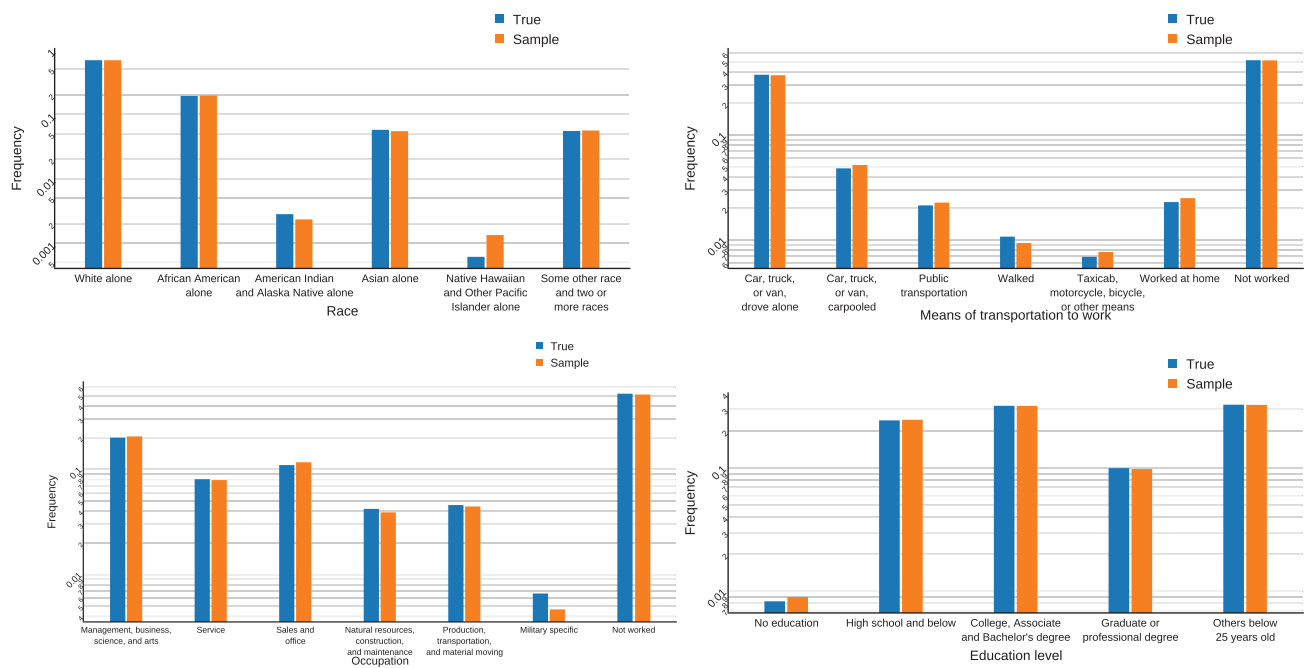

Fig. 7. Comparison of single attribute marginal distributions between the true statistics in the Virginia ACS summary data and samples generated by the categorical maximum entropy model for the attributes Race, Means of transportation to work, Occupation, and Education level. $Y$-axis is in log scale.
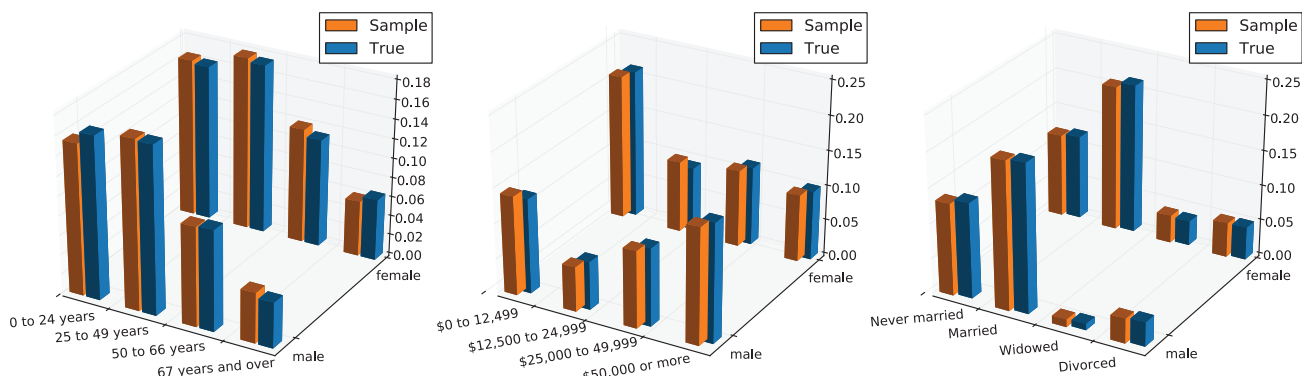

Fig. 8. Comparison of two-attribute marginal distributions between true statistics in the Virginia ACS summary data and samples generated by the categorical maximum entropy model for the categorical patterns $\{$ sex, age $\}$ (left), \{sex, income $\}$ (middle), and \{sex, marital status\} (right). For pattern \{sex, marital status\}, the pattern values whose marital status is Others under 15 years old is not displayed here since for those individuals, their marital statuses are unavailable. 


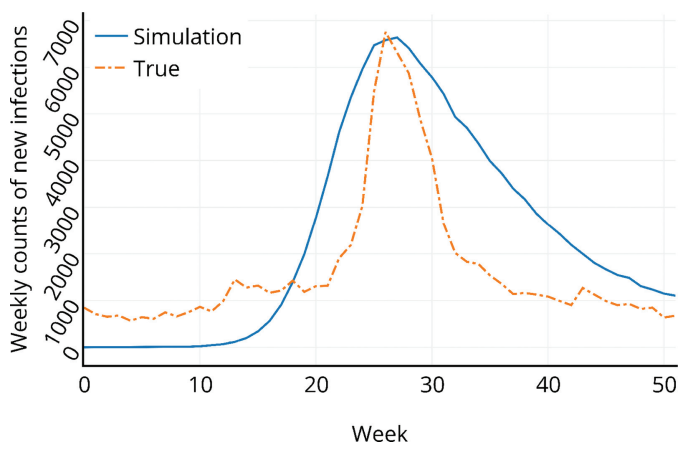

Fig. 9. The simulated weekly flu new infection counts compared to the estimated weekly new infection counts from Google Flu Trends. The simulation results are averaged across 10 independent runs.

distributions and compare them with the true distributions in the Virginia ACS summary data. We can see that the empirical distributions calculated from the synthetic individuals are very close to those in the Virginia ACS summary data. Such results demonstrate that our categorical maximum entropy model well maintains the statistical characteristics of real world datasets, and is capable of generating synthetic data for real applications.

\subsection{Application: Epidemic Simulation}

In this section, we apply our proposed categorical maximum entropy model to generate synthetic population for the city of Portland, OR in the United States, and use this model for an epidemiological simulation. We first take a synthetic contact network dataset of Portland [20] that is publicly available. The Portland dataset contains both individual demographic and contact information of residents in the city of Portland. The demographic information in this dataset contains gender, age and household income. We first group the values of age and household income into several ranges and change them into categorical features, similar to our ACS dataset analysis in Section 6.3. Then, we compute the statistics, e.g., frequencies, of the single and pairwise demographic features, convert them into categorical patterns, and infer the categorical maximum entropy model over these patterns. The Portland dataset contains 1,575,861 connected individuals, where each individual performs at least one activity with others. To generate our synthetic population, we draw 1,575,861 samples from the inferred categorical maximum entropy model.

To construct the contact network for the synthetic population, we first match the generated synthetic individuals to the real ones involved in the contact activities described in the Portland dataset based on their demographical feature values. Then, the contact network can be naturally created by connecting the synthetic individuals according to the contact activities they involve in. In this application, we choose to study the flu season in the city of Portland during the period from June 2013 to June 2014. We retrieve the estimated weekly counts of flu new infections for the city of Portland from Google Flu Trends [9], and apply the Susceptible-Infectious (SI) epidemic model over the contact network to fit the curve of weekly flu new infection counts. Figure 9 illustrates the fitted curve using the SI epidemic model. As the figure shows, the simulation results of the SI model over the synthetic population capture the trend and the peak of the weekly flu new infections in the city of Portland. These results demonstrate that the synthetic population generated by the categorical maximum entropy model is a useful model of population-level activities in cities. Here, we would like to mention that there are also many other issues, e.g., distance measuring [27, 36, 
Table 5. Comparison of Our Proposed Approach (MaxEnt) with the Existing Synthetic Population Generation Methods

\begin{tabular}{|c|c|c|c|c|c|c|c|}
\hline & IPF & FBS & PeGS & SFG & DMS & BayeNet & MaxEnt \\
\hline $\begin{array}{l}\text { Directly capture } \\
\text { dependencies beyond two } \\
\text { attributes }\end{array}$ & $\checkmark$ & & $\checkmark$ & & $\checkmark$ & $\checkmark$ & $\checkmark$ \\
\hline $\begin{array}{l}\text { Directly estimate } \\
\text { population joint } \\
\text { distribution }\end{array}$ & & & & $\checkmark$ & & $\checkmark$ & $\checkmark$ \\
\hline $\begin{array}{l}\text { Don't require seeding } \\
\text { population }\end{array}$ & & & $\checkmark$ & & & $\checkmark$ & $\checkmark$ \\
\hline $\begin{array}{l}\text { Support iterative model } \\
\text { inference }\end{array}$ & & & & & & & $\checkmark$ \\
\hline
\end{tabular}

37], that are relevant when studying disease transmission. However, these topics are beyond the scope of this article, thus, we will not discuss them in detail here.

\section{RELATED WORK}

The problem of generating synthetic data that maintain the structures and dependencies in actual data has been studied by researchers from various realms. Iterative proportional fitting (IPF) technique [2] and its variants [18, 38], which generally operate over contingency tables, have been applied to generate synthetic population to study large social contact networks, land use, and transportation microsimulation. The NDSSL at Virginia Tech released synthetic datasets of population in the city of Portland [20] generated by a high-performance simulation system Simfrastructure which adopts IPF techniques. Such IPF-based approaches usually do not directly estimate the joint probability distribution of the data, and sometimes, they require seeding populations as a part of the input. Fitness-based synthesis (FBS) approaches [15] define fitness measures based on control tables to directly generate synthetic populations with seeding data. Sample-free generators (SFG) [3] were proposed to generate synthetic populations using the joint data distribution defined with the data at the most disaggregated level Namazi-Rad et al. [19] applied a dynamic micro-simulation model (DMS) to project dynamics over the synthetic population generated by combinational optimization approaches. Recently, a non-parametric perturbed Gibbs sampler (PeGS) [21] that requires estimating all of the full conditional distributions to represent the joint data distribution was proposed to generate large-scale privacy-safe synthetic health data. Sun and Erath [26] proposed a Bayesian network (BayeNet) based approach to estimate the joint distribution of populations, which is then used to generate synthetic populations. While, our proposed maximum entropy model additionally supports iterative model inference, which makes it easy to update our proposed model with new knowledge about the data. Compared to the proposed categorical maximum entropy model, these existing approaches described above either do not directly capture the dependencies beyond two attributes or do not directly estimate the full joint data distribution. Table 5 compares the proposed maximum entropy approach (MaxEnt) with the related existing synthetic population generation methods in detail.

Maximum entropy models have drawn much attention recently in the pattern mining community, especially in the realm of discovering subjectively interesting patterns. De Bie [7] formalized an information theoretical framework for data mining by applying the maximum entropy principle. In recent research works $[8,23,32]$, maximum entropy models were developed to discover and 
evaluate interesting patterns from binary datasets, and they were also adopted together with the principle of Minimum Description Length to summarize and compress binary datasets [16, 31, 34]. Besides the binary data, maximum entropy modeling was also extended to the multi-relational data and real-valued data [14, 24, 35]. However, all these previous works focus on discovering informative patterns or assessing subjective interestingness of patterns from binary, real-valued, or multi-relational data, and none of them involves categorical data.

In the database community, Gray et al. [10] surveyed several database generation techniques that generate large scale synthetic datasets, and Bruno and Chaudhuri [4] proposed a Data Generation Language that allows individual attribute distributions to be specified. A database generation tool that could handle complex inter- and intra-table relationships was proposed by Houkjær et al. [11]. Arasu et al. [1] proposed an efficient, linear programming based algorithm to generate synthetic relational databases that satisfy a given set of declarative constraints. Compared to the proposed approach, these works focus on structured data in relational databases, while our proposed method is generally applicable to categorical data including unstructured categorical data. The maximum entropy principle is also adopted in database query optimization. The sizes of database queries were estimated by modeling complicated database statistics using maximum entropy probability distributions [13, 17]. Ré and Suciu [22] studied the problem of cardinality estimation using the entropy maximization technique with peak approximation. An algorithm called ISOMER was proposed by Srivastava et al. [25] to approximate the true data distribution by applying the maximum entropy principle over database query feedbacks. These works aim to utilize the maximum entropy principle to optimize database queries, while in our method, we are focusing on estimating a probabilistic generative model so that synthetic data could be generated.

\section{CONCLUSION}

In this article, we have demonstrated a generative probabilistic model for categorical data by employing the maximum entropy principle. By introducing categorical tuple blocks and the corresponding partial order over them, we have presented an efficient model inference algorithm based on the well-known iterative scaling framework. Experiment results on both synthetic data and real US census data show that the proposed model well estimates the underlying categorical data distributions. The application to the problem of epidemic simulation demonstrates that our proposed model can be applied to support research in a variety of application areas.

\section{ACKNOWLEDGMENTS}

The US Government is authorized to reproduce and distribute reprints of this work for Governmental purposes notwithstanding any copyright annotation thereon. Disclaimer: The views and conclusions contained herein are those of the authors and should not be interpreted as necessarily representing the official policies or endorsements, either expressed or implied, of IARPA, DoI/NBC, NSF, Army Research Laboratory, or the U.S. Government.

\section{REFERENCES}

[1] Arvind Arasu, Raghav Kaushik, and Jian Li. 2011. Data generation using declarative constraints. In Proceedings of the SIGMOD’11. 685-696.

[2] C. L. Barrett, R. J. Beckman, M. Khan, V. Kumar, M. V. Marathe, P. E. Stretz, T. Dutta, and B. Lewis. 2009. Generation and analysis of large synthetic social contact networks. In Proceedings of the WSC. 1003-1014.

[3] Johan Barthelemy and Philippe L. Toint. 2013. Synthetic population generation without a sample. Transportation Science 47, 2 (2013), 266-279.

[4] Nicolas Bruno and Surajit Chaudhuri. 2005. Flexible database generators. In Proceedings of the VLDB'05. VLDB Endowment, 1097-1107. 
[5] Imre Csiszár. 1975. I-divergence geometry of probability distributions and minimization problems. Annals of Probability 3, 1 (1975), 146-158.

[6] J. N. Darroch and D. Ratcliff. 1972. Generalized iterative scaling for log-linear models. The Annals of Mathematical Statistics 43, 5 (1972), 1470-1480.

[7] Tijl De Bie. 2011. An information theoretic framework for data mining. In Proceeding of the KDD'11. ACM, 564-572.

[8] Tijl De Bie. 2011. Maximum entropy models and subjective interestingness: An application to tiles in binary databases. Data Mining and Knowledge Discovery 23, 3 (Nov. 2011), 407-446.

[9] Google Inc. 2014. Data Source: Google Flu Trends. Retrieved from http://www.google.org/flutrends.

[10] Jim Gray, Prakash Sundaresan, Susanne Englert, Ken Baclawski, and Peter J. Weinberger. 1994. Quickly generating billion-record synthetic databases. In Proceedings of the SIGMOD'94. ACM, 243-252.

[11] Kenneth Houkjær, Kristian Torp, and Rico Wind. 2006. Simple and realistic data generation. In Proceedings of the VLDB'06. 1243-1246.

[12] W. Hu, L. Yan, and H. Wang. 2014. Traffic jams prediction method based on two-dimension cellular automata model. In Proceedings of the ITSC. 2023-2028.

[13] Raghav Kaushik, Christopher Ré, and Dan Suciu. 2009. General database statistics using entropy maximization. In Proceedings of the DBPL'09. Springer-Verlag, 84-99.

[14] Kleanthis-Nikolaos Kontonasios, Jilles Vreeken, and Tijl De Bie. 2013. Maximum entropy models for iteratively identifying subjectively interesting structure in real-valued data. In Proceedings of the ECMLPKDD'13. Springer, 256-271.

[15] Lu Ma and Sivaramakrishnan Srinivasan. 2015. Synthetic population generation with multilevel controls: A fitnessbased synthesis approach and validations. Computer-Aided Civil and Infrastructure Engineering 30, 2 (2015), 135-150.

[16] Michael Mampaey, Nikolaj Tatti, and Jilles Vreeken. 2011. Tell me what I need to know: Succinctly summarizing data with itemsets. In Proceedings of the KDD'11. ACM, 573-581.

[17] V. Markl, N. Megiddo, M. Kutsch, T. M. Tran, P. Haas, and U. Srivastava. 2005. Consistently estimating the selectivity of conjuncts of predicates. In Proceedings of the VLDB'05. 373-384.

[18] Kirill Mueller and Kay W. Axhausen. 2011. Hierarchical IPF: Generating a synthetic population for switzerland. In ERSA Conference Papers. European Regional Science Association.

[19] Mohammad-Reza Namazi-Rad, Payam Mokhtarian, and Pascal Perez. 2014. Generating a dynamic synthetic population-Using an age-structured two-sex model for household dynamics. PLoS One 9, 4 (2014).

[20] Network Dynamics and Simulation Science Laboratory. 2015. Synthetic Data Products for Societal Infrastructures and Proto-Populations: Data Set 2.0. Technical Report. Virginia Polytechnic Institute and State University. NDSSL-TR-07003.

[21] Y. Park, J. Ghosh, and M. Shankar. 2013. Perturbed gibbs samplers for generating large-scale privacy-safe synthetic health data. In Proceedings of the ICHI'13. 493-498.

[22] Christopher Ré and Dan Suciu. 2010. Understanding cardinality estimation using entropy maximization. In Proceedings of the PODS'10. ACM, 53-64.

[23] Koen Smets and Jilles Vreeken. 2012. SLIM: Directly mining descriptive patterns. In Proceedings of the SDM'12. SIAM, 236-247.

[24] Eirini Spyropoulou, Tijl De Bie, and Mario Boley. 2014. Interesting pattern mining in multi-relational data. Data Mining and Knowledge Discovery 28, 3 (2014), 808-849.

[25] U. Srivastava, P. J. Haas, V. Markl, M. Kutsch, and T. M. Tran. 2006. ISOMER: Consistent histogram construction using query feedback. In Proceedings of the ICDE'06. IEEE Computer Society.

[26] Lijun Sun and Alexander Erath. 2015. A bayesian network approach for population synthesis. Transportation Research Part C: Emerging Technologies 61 (2015), 49-62.

[27] M. Tan, B. Wang, Z. Wu, J. Wang, and G. Pan. 2016. Weakly supervised metric learning for traffic sign recognition in a LIDAR-equipped vehicle. IEEE Transactions on Intelligent Transportation Systems 17, 5 (May 2016), 1415-1427.

[28] D. Tao, X. Li, X. Wu, and S. J. Maybank. 2007. General tensor discriminant analysis and gabor features for gait recognition. IEEE Transactions on Pattern Analysis and Machine Intelligence 29, 10 (2007), 1700-1715.

[29] D. Tao, X. Li, X. Wu, and S. J. Maybank. 2009. Geometric mean for subspace selection. IEEE Transactions on Pattern Analysis and Machine Intelligence 31, 2 (2009), 260-274.

[30] Nikolaj Tatti. 2006. Computational complexity of queries based on itemsets. Information Processing Letters 98, 5 (2006), 183-187.

[31] Nikolaj Tatti and Jilles Vreeken. 2008. Finding good itemsets by packing data. In Proceedings of the ICDM'08. IEEE, 588-597.

[32] Nikolaj Tatti and Jilles Vreeken. 2011. Comparing apples and oranges: Measuring differences between data mining results. In Proceeding of the ECMLPKDD'11. Springer, 398-413.

[33] United States Census Bureau. 2012. American Community Survey. Retrieved from http://www.census.gov/acs/www/. 
[34] Jilles Vreeken, Matthijs van Leeuwen, and Arno Siebes. 2011. KrimP: Mining itemsets that compress. Data Mining and Knowledge Discovery 23, 1 (2011), 169-214.

[35] Hao Wu, Jilles Vreeken, Nikolaj Tatti, and Naren Ramakrishnan. 2014. Uncovering the plot: Detecting surprising coalitions of entities in multi-relational schemas. Data Mining and Knowledge Discovery 28, 5-6 (2014), 1398-1428.

[36] J. Yu, Y. Rui, Y. Y. Tang, and D. Tao. 2014. High-order distance-based multiview stochastic learning in image classification. IEEE Transactions on Cybernetics 44, 12 (2014), 2431-2442.

[37] J. Yu, X. Yang, F. Gao, and D. Tao. 2017. Deep multimodal distance metric learning using click constraints for image ranking. IEEE Transactions on Cybernetics PP, 99 (2017), 1-11.

[38] Yi Zhu and Joseph Ferreira. 2014. Synthetic population generation at disaggregated spatial scales for land use and transportation microsimulation. Journal of the Transportation Research Board 2429, 1 (2014), 168-177.

Received February 2017; revised October 2017; accepted January 2018 